\title{
Important notes:
}

You MUST use this template. If you don't use it your abstract WILL be rejected.

Do NOT enter author and institution information on this form. You will be able to enter this information online when you submit the abstract.

Do NOT write outside the boxes. Any text or images outside the boxes will be deleted.

Do NOT alter this form by deleting parts of it or adding new boxes. Simply enter your information into the boxes. We use automatic processing - if you alter it your submission will not be processed correctly.

Save this file in .doc or .docx format.

Title:

Gait symmetry in walking and running after unilateral total knee replacement: Is there a relationship between walking and running symmetry?

\section{Abstract: (Your abstract must use 10 point New Times Roman style and must fit into the box. Do not enter author details)}

Introduction: Gait symmetry is a characteristic of interest in unilateral conditions, such as hip arthroplasty and it has been suggested that this can lead to gait asymmetry [1]. Gait symmetry is increasingly assessed after stroke [2] and is a measure of motor control in walking to inform treatment [3]. However, the use of gait symmetry transfers to other unilateral conditions and has been used after ankle arthroplasty [4]. Therefore, it is assumed it can also be used after total knee replacement (TKR). The aim of this study is to investigate if the symmetry ratios (SR) reported in TKR participants whilst walking, correlate to the SR whilst running. This contributes to a larger investigation currently being conducted by the authors on a new generation of TKR, involving more demanding activities not previously assessed in this patient group (such as running).

Research Question: Are symmetry ratios of TKR participants related between walking and running?

Methods: NHS ethical approval (15/SC/0725) was gained for this study. Six (4 female, 2 male) TKR participants implanted with the Physica KR TKR (LimaCorporate, Italy), a minimum of 12 months post-operative were recruited.

Gait analysis was performed using a 3D 10 camera motion capture system

(VICON, Oxford), synchronised with two Kistler force plates. Marker data was sampled at $250 \mathrm{~Hz}$ and force data at $2000 \mathrm{~Hz}$. Six successful trials were recorded and three were selected for processing once reviewed. Three-trial averages were used for each parameter (V), and then SR was calculated. The parameters investigated included peak knee flexion (PKF), peak knee extension (PKE), range of motion (ROM), swing time and stance time.

Symmetry ratio $(\mathrm{SR})=\mathrm{V}_{\mathrm{op}} / \mathrm{V}_{\text {non-op }}$

Perfect gait symmetry equates to 1 . Pearson's correlation coefficients determined relationship for the SR (for each parameter) between walking and running.

Results:

\begin{tabular}{|c|c|c|c|c|c|c|}
\hline & & $\operatorname{PKF}\left({ }^{\circ}\right)$ & PKE $\left(^{\circ}\right)$ & $\operatorname{ROM}\left({ }^{\circ}\right)$ & Swing time (s) & Stance time (s) \\
\hline \multirow[t]{3}{*}{ Walking } & Op & 43.94 & -3.50 & 47.44 & 0.48 & 0.72 \\
\hline & Non-op & 40.26 & -4.58 & 44.84 & 0.47 & 0.71 \\
\hline & SR & 1.27 & -0.32 & 1.10 & 0.98 & 1.02 \\
\hline \multirow[t]{3}{*}{ Running } & Op & 69.97 & 2.66 & 67.04 & 0.42 & 0.31 \\
\hline & Non-op & 68.69 & -1.03 & 68.53 & 0.40 & 0.33 \\
\hline & SR & 1.02 & 0.87 & 1.00 & 1.04 & 0.95 \\
\hline
\end{tabular}

Discussion: A symmetry ratio of 1 would denote perfect symmetry between the operated and non-operated leg. It was found that many of the SR values were close to perfect symmetry, implying that the TKR performs in a similar way to the healthy knee. This in turn suggests positive results from the TKR surgery. There were no significant correlations between the results reported whilst walking and running, therefore there is no relationship in the symmetry ratio knee kinematics and gait phases for walking and running in TKR patients.

References:

[1] Patterson et al., Gait Posture 2012; 35: 590-594. [2] Patterson et al., Gait Posture 2010; 31: 241-246. [3] Patterson et al., Arch Phys Med Rehabil 2008; 89: 304-310. [4] Ledoux et al., Foot and Ankle Surgery 2016; 22: 115. 